\title{
Model Development for Transport Studies in Negative Shear Modes
}

\author{
M.C. Spang, T. Casper, B. Stallard, K.I. Thomassen
}

May, 1997

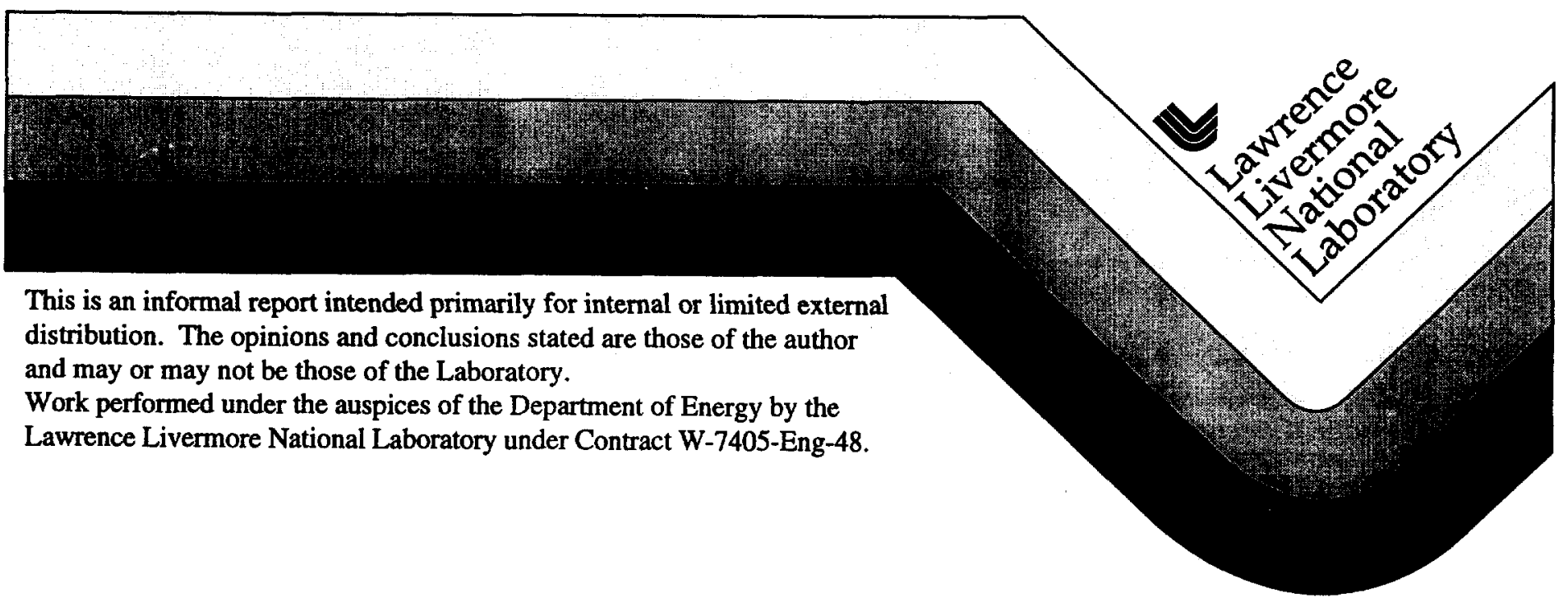




\section{DISCLAIMER}

This document was prepared as an account of work sponsored by an agency of the United States Government. Neither the United States Government nor the University of Califormia nor any of their employees, makes any warranty, express or implied, or assumes any legal liability or responsibility for the accuracy, completeness, or usefulness of any information, apparatus, product, or process disclosed, or represents that its use would not infringe privately owned rights. Reference herein to any specific commercial product, process, or service by trade name, trademark, manufacturer, or otherwise, does not necessarily constitute or imply its endorsement, recommendation, or favoring by the United States Government or the University of California. The views and opinions of authors expressed herein do not necessarily state or reflect those of the United States Government or the University of California, and shall not be used for advertising or product endorsement purposes.

This report has been reproduced directly from the best available copy.

Available to DOE and DOE contractors from the

Office of Scientific and Technical Information

P.O. Box 62, Oak Ridge, TN 37831

Prices available from (615) 576-8401, FTS 626-8401

Available to the public from the

National Technical Information Service

U.S. Department of Commerce

5285 Port Royal Rd.

Springfield, VA 22161 
Model Development for Transport Studies in Negative Shear Modes

M. C. Spang, T. Casper, B. Stallard, and K. I. Thomassen Lawrence Livermore National Laboratory

May 1997 


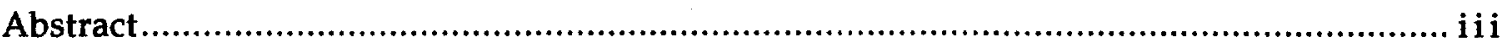

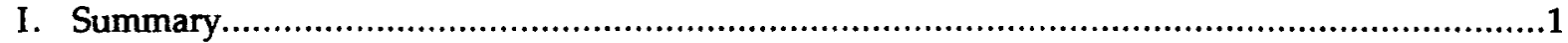

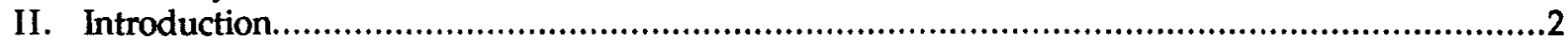

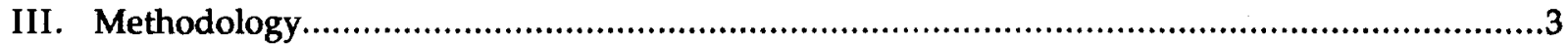

IV. Results and Conclusions........................................................................................................9

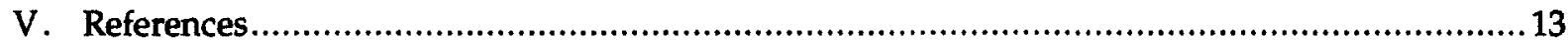

\section{List of Figures}

Figure II.1

Figure II.2

Figure II.3

Figure II.4

Figure III.1

Figure III.2

Figure III.3

Figure III.4

Figure IV.1

(a) Comparison of RLW and TRANSP $\chi_{e}$ vs. $\sqrt{\psi}$

(b) Comparison of RLW and TRANSP $\chi_{1}$ vs. $\sqrt{\psi}$

(c) Comparison of RLW and TRANSP D vs. $\sqrt{\psi}$

Figure IV.2

(a) Comparison of RLW and Experimental Electron Temperature Profiles

(b) Comparison of RLW and Experimental Ion Temperature Profiles....

Figure IV.3

(a) Comparison of RLW and Experimental Safety Factor Profiles.

(b) Comparison of RLW and Experimental Total Current Density Profiles.

Figure IV.4

Plasma Current and Neutral Beam Power vs. Time. 3

(b) Experimental Ion Density Profiles vs. $\sqrt{\psi}$. .4

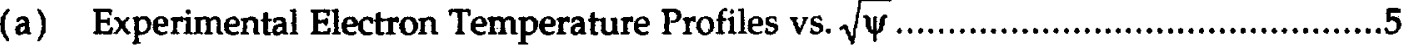

(b) Experimental Ion Temperature Profiles vs. $\sqrt{\psi}$ .5

TRANSP Transport Coefficient Profiles at 1.38 Seconds ......................................7 Comparison of Unadjusted and Adjusted $\chi_{\mathrm{i}}$ at 1.38 Seconds...................................8

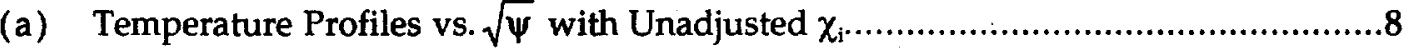

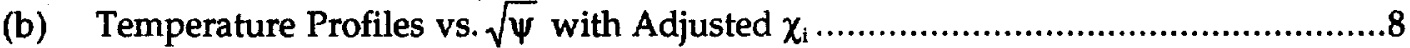

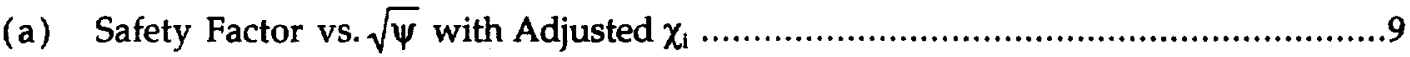

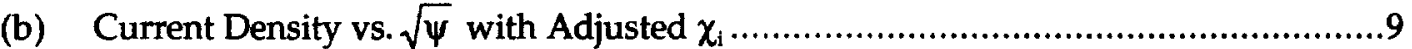

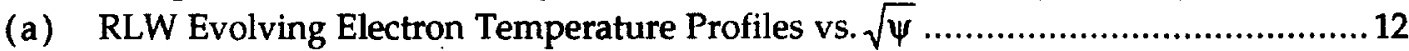

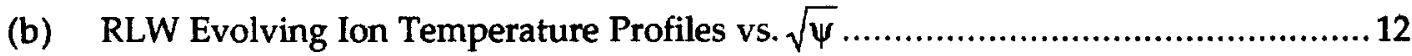


This study develops a simple transport model which can be used predictively for tokamak negative central shear (NCS) modes, with the assistance of Lawrence Livermore National Laboratory's core plasma simulation code, CORSICA ${ }^{13}$. The results show that the the Rebut-Lallia-Watkins Critical Electron Temperature Gradient Model, coupled with an NCS transport model and TRANSP ${ }^{14}$ data, renders a reasonably close match to experimental temperature profiles. Additionally, this research offers the first benchmark calculation indicating that the CORSICA code itself, when given transport coefficients from the analysis of experimental data, replicates the experimental profiles, indicating that both TRANSP and CORSICA together are consistent in their analysis of the plasma evolution. This means CORSICA is working properly and has no known major internal flaws. 


\section{SUMMARY}

The purpose of this project was to develop a transport model that could be used predictively for a class of modes known as negative central shear (NCS) modes in tokamaks, and to also test a specific plasma transport model developed by Rebut, Lallia, and Watkins (RLW) [1]. The interest is in applying the model to "what if" scenarios in the DIII-D tokamak to determine optimum operating regimes and then subsequently testing them in order to compare theory and experiment. The transport model was incorporated into the Lawrence Livermore National Laboratory (LLNL) core plasma simulation code CORSICA [13]. This code simulates the evolution of plasma profiles, such as density, temperature, current density, and safety factor in a tokamak.

The RLW model was developed under the premise that magnetic turbulence is largely responsible for anomalous transport and that this transport is triggered when the electron temperature gradient surpasses a critical value. By using dimensional analysis, the RLW model provides an expression for the critical electron temperature gradient which stepwise switches on intensified (above neoclassical levels) thermal and particle transport when exceeded.

LLNL is interested in incorporating several different transport models into CORSICA to evaluate proposed tokamak scenarios. CORSICA is written using the BASIS language, which allows many plasma codes to interact during their execution. This is an effective way to couple and simultaneously solve equilibrium, stability, transport, current drive, heating, and other plasma phenomenology which are all mutually dependent. The code contains a free boundary equilibrium solver and, when coupled to a transport package written in toroidal flux coordinates, it evolves 2-D equilibria consistent with both transport and Grad-Shafranov equilibrium constraints. It additionally contains a neutral beam package which characterizes sources of particles, heat, and current.

This study concentrates on developing a transport model for the negative central shear mode of operation in tokamaks, an enhanced confinement state in which transport is reduced to neoclassical levels. NCS results from a hollow current density profile as opposed to a profile which is peaked onaxis, the normal mode for tokamak operation, and, as its name suggests, is characterized by negative shear $(S=r \nabla q / q)$ over the central region of the plasma. The safety factor, $q(r)$, is related to the pitch of the magnetic field lines relative to the toroidal axis.

To motivate interest in high-performance modes, note that velocity shear (such as changes in the ExB velocity with radius) can tear apart the magnetic islands that would normally grow when ion temperature gradient modes develop. Such shear is observed near the edge of the plasma, and transport barriers are consequently created there. This gives rise to the so-called H-mode (high-mode) of confinement in the plasma, reducing transport in the core. More recently, it has also been observed that in regions of negative magnetic shear the levels of transport in that region are even lower, as low as allowed from neoclassical theory. Thus an additional transport barrier forms at the radius where the shear parameter is zero, and transport inside that radius is reduced. Since the RLW model is valid only in regions of positive shear, a NCS transport model was heuristically developed and then added to the RLW model thereby constructing a set of complete core transport profiles.

This study analyzes the NCS shot 84682, obtained from the DIII-D experiment located at General Atomics in San Diego, California. The results were extremely interesting, and the model proved to be workable for the predictive studies that LLNL is now pursuing. Transport coefficients calculated from this model reasonably match those obtained experimentally from the reference shot at the point $S=0$, although the errors grow large as the edge of the plasma is approached. Further, the RLW coefficients were two orders of magnitude larger than those predicted by standard neoclassical theory, indicating that they largely model anomalous turbulent transport in the positive shear region. 


\section{INTRODUCTION}

It was discovered very early on that plasma transport does not follow classical processes determined only by collisions of particles in their orbits, but has an additional component termed "anomalous". In the case of toroidal devices with complex particle motions (such as banana orbits, axis-encircling orbits, etc.) that differ according to the regime of collisionality, transport by collisions is called neoclassical transport (see [12] for discussion). Anomalous transport can be quite large, especially for electrons, and can scale unfavorably with the magnetic field i.e., $\mathrm{B}^{-1}$ as in Bohm diffusion rather that $\mathrm{B}^{-2}$ as classically determined. Furthermore, discovery of the underlying physics of the turbulent processes at play has eluded several decades of researchers. If transport could be reduced to its neoclassical levels, fusion performance in a given device would be considerably enhanced. For these reasons, the development of plasma transport models is a current and very high priority topic of research in magnetic fusion.

Ion temperature gradient (ITG) modes are now widely thought to be responsible for the anomalies of ion transport, and a number of researchers are incorporating these modes into non-linear models that evolve this instability to its saturation levels. Estimates of the transport resulting from these turbulent fluctuations can be made using the growth rate and wavenumber spectra of the turbulence. In contrast, RLW treats the anomalies in electron transport and assumes that the ions follow suit in a prescribed fashion.

The RLW model, as initially described [1, 2], was further refined [3] in 1992 and is the version currently used in CORSICA. The RLW model was developed under the premise that magnetic turbulence is largely responsible for anomalous transport and that this transport is triggered when the electron temperature gradient surpasses a critical value. It is analogous to fluid theory which has found that when fluid flow exceeds a critical dimensionless parameter, namely the Reynolds number, transition from laminar to turbulent flow will ensue. Similarly, the RLW model proposes that when a critical electron temperature gradient is exceeded, anomalous transport will be present in addition to neoclassical transport, except in regions of negative central shear $(\nabla \mathrm{q}<0$, where $\mathrm{q}$ is the safety factor). An anomalous transport coefficient $\chi^{\mathrm{AN}}$ is introduced in a stepwise fashion if $\left|\nabla T_{e}\right|>\left|\nabla T_{e, c r t t}\right|$ and added to $\chi^{\text {neo }}$.

The particle and heat fluxes ( $\Gamma$ and $q$, respectively) are determined from gradients in density and temperature by $\Gamma_{e, i}=-D_{e, i} \nabla n_{e, i}$ and $q_{e, i}=-n_{e, i} \chi_{e, i} \nabla T_{e, i}$ and used in the coupled two-fluid continuity, momentum, and energy equations of CORSICA. However, it should be noted that CORSICA uses only $D_{i}$ and treats the electrons by demanding quasi-neutrality. These equations are 1-D in the toroidal flux coordinate, while the time-dependent equilibrium is 2-D in order to allow different poloidal shapes. As stated earlier, this simulation code allows interactive access to all code parameters.

If the critical electron gradient is exceeded, anomalous transport is assumed to be active by the RLW model, and the following anomalous RLW transport coefficients are added to the neoclassical coefficients:

$$
\begin{aligned}
& \chi_{e}^{\mathrm{AN}}=\mathrm{C}_{e}^{\mathrm{RLW}}\left(1-\left|\frac{\nabla \mathrm{T}_{e}^{\mathrm{crit}}}{\nabla \mathrm{T}_{e}}\right|\right) \mathrm{H}(\nabla \mathrm{q}) \mathrm{H}\left(\left|\nabla \mathrm{T}_{e}\right|-\left|\nabla \mathrm{T}_{e, \text { crit }}\right|\right) \\
& \chi_{1}^{\mathrm{AN}}=\chi_{\mathrm{e}}^{\mathrm{AN}} \sqrt{\frac{\mathrm{T}_{e}}{\mathrm{~T}_{1}}} \frac{2 Z_{\mathrm{i}}}{\sqrt{1+Z_{\text {eff }}}} \\
& D_{e}^{A N}=D_{i}^{A N}=D^{A N}=\alpha \chi_{e}^{A N}
\end{aligned}
$$

where $\mathrm{H}$ indicates the Heaviside function, $\mathrm{c}_{e}^{\mathrm{RLW}}$ is the RLW multiplier given in [3], and $\alpha$ is a proportionality constant set to 1.0. It is beyond the scope of this project, and indeed of the state of the art in the field, to model transport coefficients of all species correctly, so we have the limited objective of incorporating the RLW model into CORSICA to use in proposing NCS scenarios in DIII-D. CORSICA uses Chang-Hinton [4] neoclassical transport coefficients. 
Now, in order to construct composite $\chi_{e}, \chi_{i}$ and D profiles for the entire plasma, models are needed for the NCS region. To develop these models, the Princeton Plasma Physics Laboratory's (PPPL) transport code TRANSP [14] was used in analysis mode on the experimental data in order to deduce values of electron and ion diffusivities throughout the NCS and positive shear regions. Unfortunately TRANSP currently yields inaccurate $\chi_{1}$ near the axis as discussed in the next section, so a constant value of $\chi_{\mathrm{i}}$ was determined by iteratively using CORSICA and matching the resulting profiles to experiment. Subsequently, these NCS models were melded with the positive shear RLW models to construct total core transport profiles.

The experimental data analyzed in this study is from DIII-D NCS shot 84682, shown below in Figures II.1 to II.4. Figure II.1 shows the total plasma current and neutral beam power as functions of time. This study concentrates on the time range 1.2 to 1.4 seconds, the portion of the shot in which the plasma current has nearly leveled off to 1.6 MA and the NCS configuration is formed. Figures II.2 and II. 3 show the radial profiles, in $\sqrt{\psi}$-coordinates, of density and temperature for both electrons and ions. Here, $\psi$ is the toroidal flux coordinate. It should be repeated that the experimental density profiles were used throughout this study, while the temperature profiles were evolved.

Figure II.1

Plasma Current and Neutral Beam Power versus Time

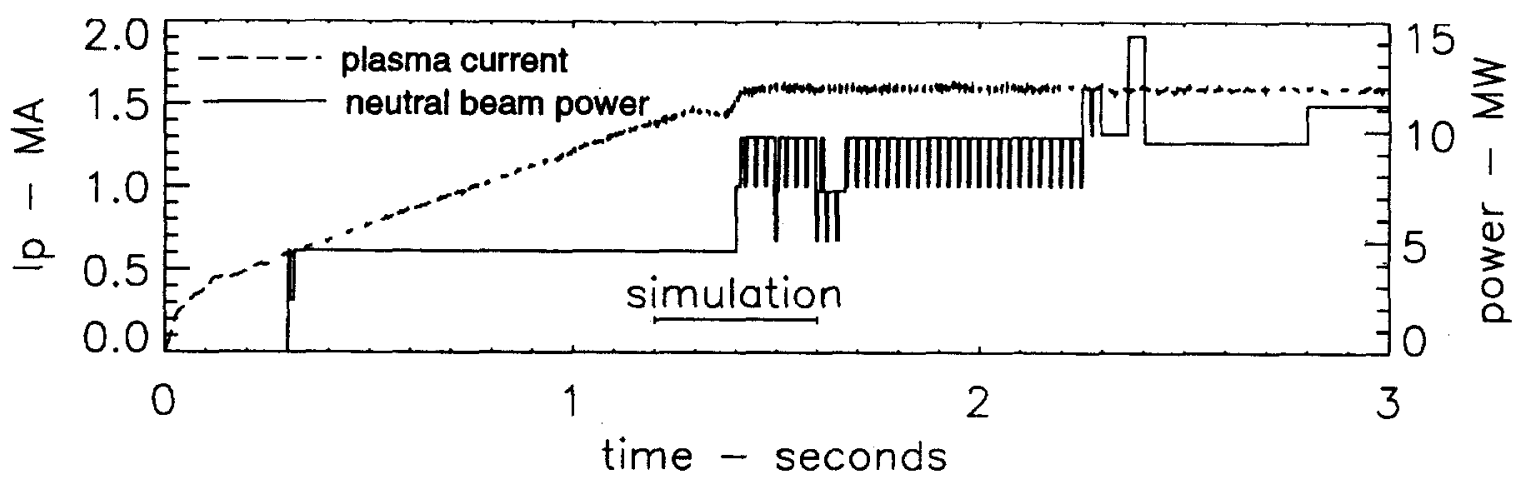

By using the experimental profiles for both density and temperature, the code gives the safety factor, $q$, as well as the profiles for the neutral beam (using a Monte Carlo simulation), bootstrap, and ohmic current densities. The q-profile and total current density are shown in Figure II.4. As seen from the q-profile, the NCS region begins at approximately $\sqrt{\psi}=0.6$ at 1.2 seconds and moves inward as time progresses to $\sqrt{\psi}=0.52$ at 1.4 seconds. The current density profile is hollow, the hallmark. of a NCS shot.

\section{METHODOLOGY}

The first step in creating a composite model is to determine an appropriate value for $\chi_{1}$ in the NCS region. Experimentally, it is known that the coefficient is close to its neoclassical value. In fact, the first data [5; 10] from both DIII-D and TFTR gave values below those calculated from neoclassical theory. This of course is in error, since the neoclassical value sets an absolute minimum. The difficulty is in the approximations used to describe the particle orbits, which are fundamental to a determination of the transport coefficients. From simple theory it is understood that collisions cause a random walk step away from the orbit, and the coefficients are related to the size of that step and the collision frequency.

It turns out that models of the orbits are not valid in the core of the plasma where gradient lengths are large. Specifically, in the NCS region the ion poloidal gyroradius can be comparable in magnitude, or even greater than, the local minor radius and/or the equilibrium pressure gradient scale length. These conditions violate assumptions of the calculations of $\chi_{i}$. Recent work by Lin et al. [11] describes 
Figure II.2

(a)

Experimental Electron Density Profiles

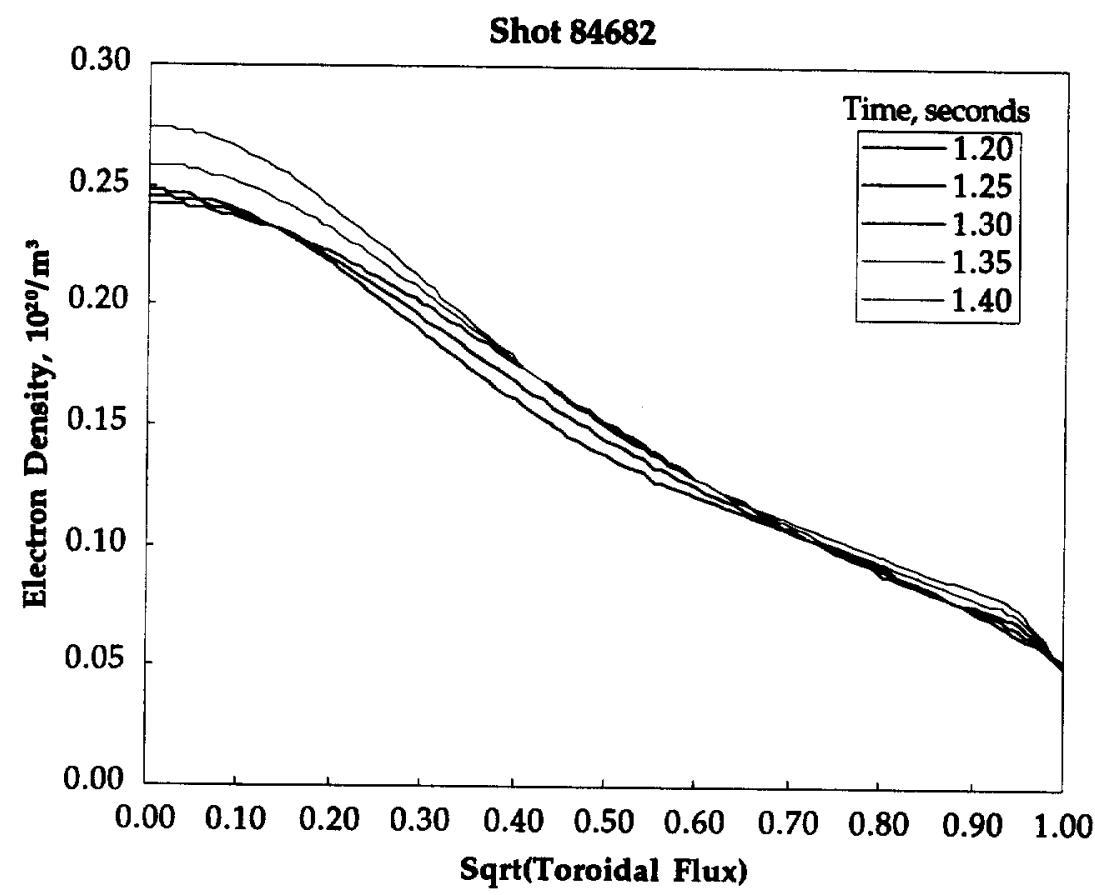

(b)

Experimental Ion Density Profiles

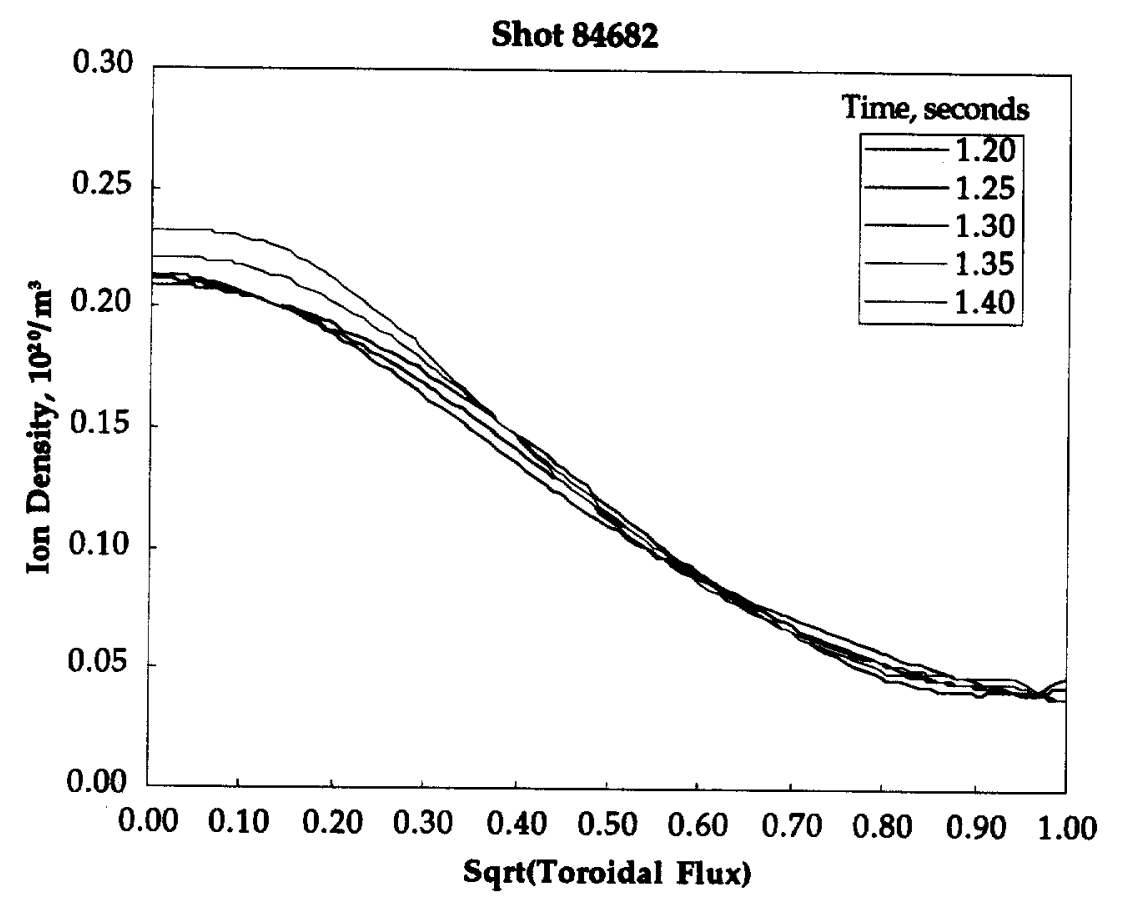


Figure II.3

(a)

Experimental Electron Temperature Profiles

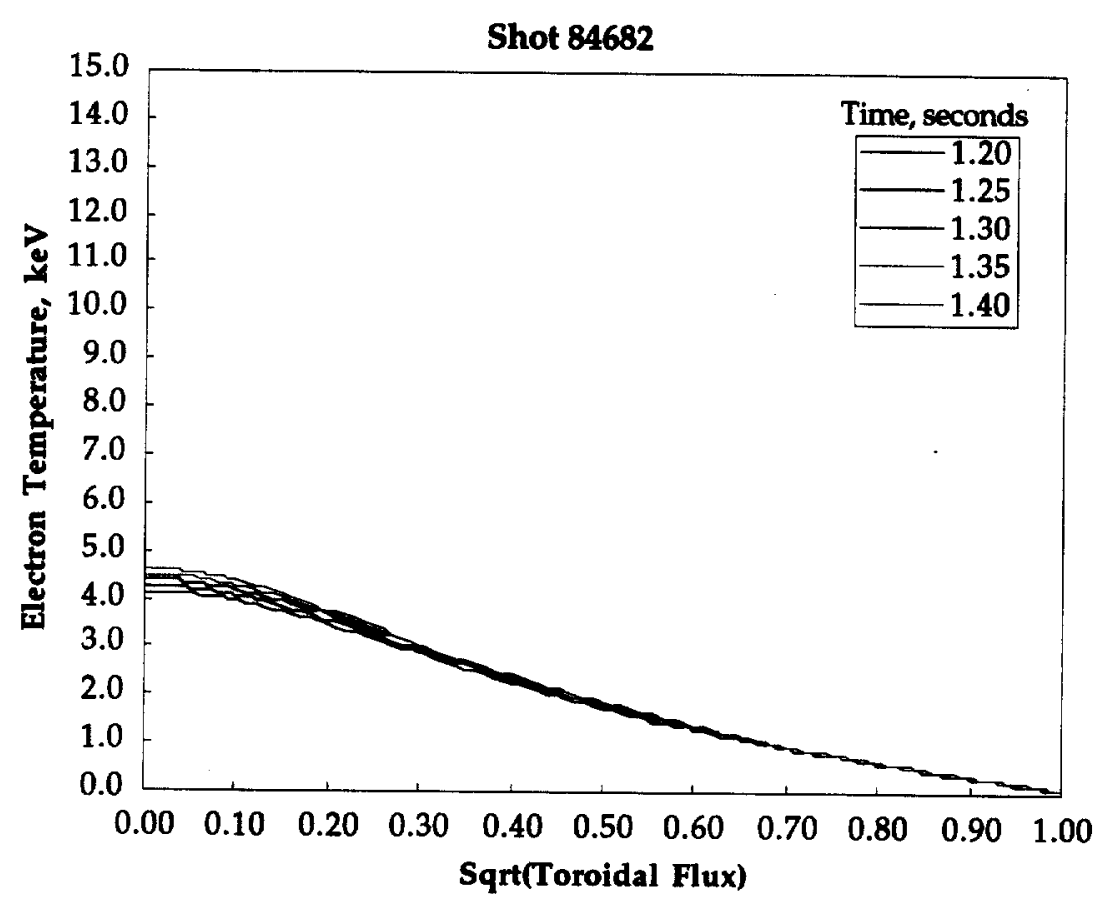

(b)

Experimental Ion Temperature Profiles

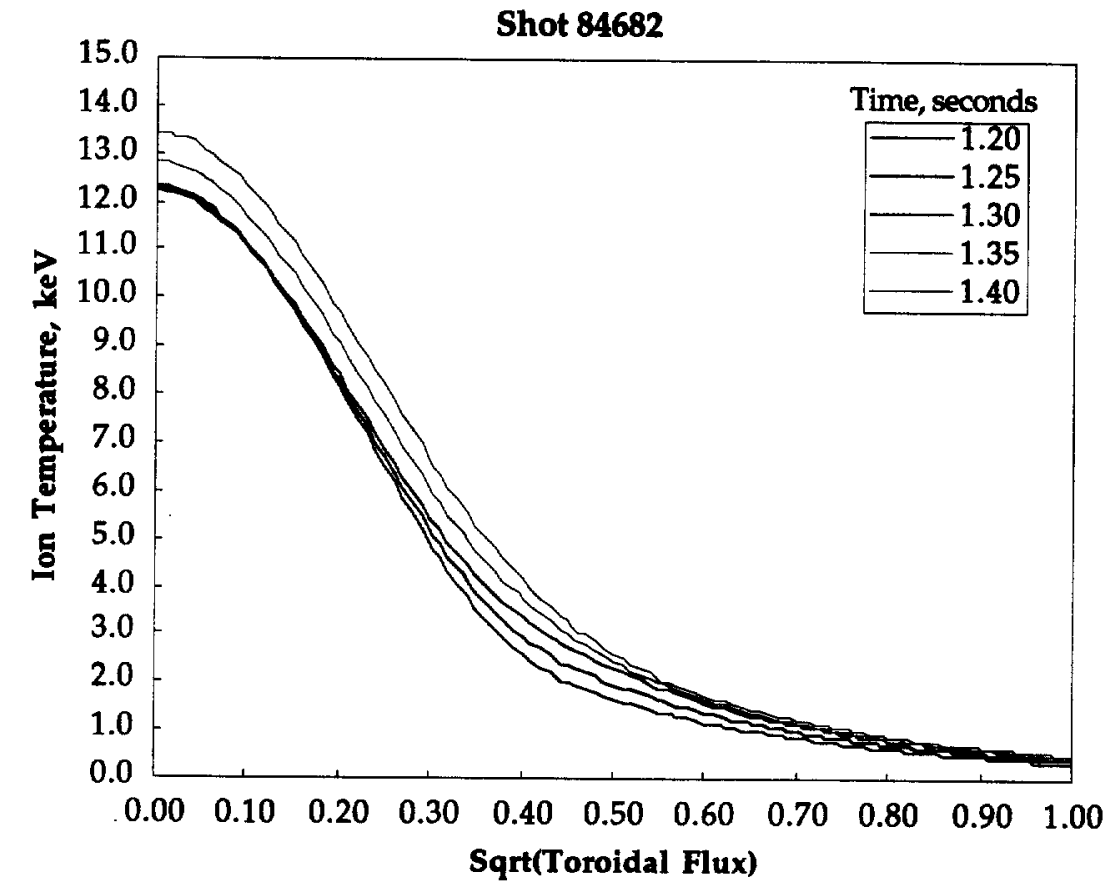




\section{Figure II.4}

(a)

Safety Factor Profile Using Experimental Density and Temperatures

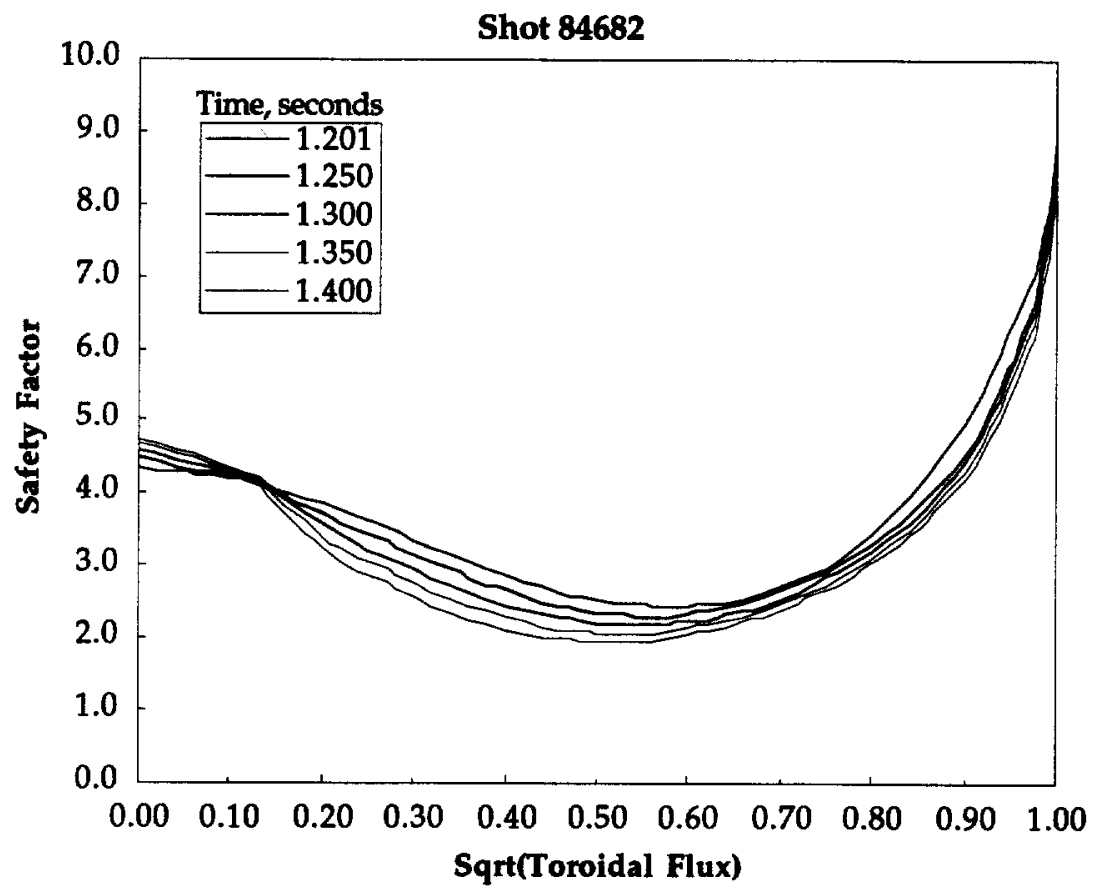

(b)

Total Current Density Profile Using Experimental Density and Temperatures

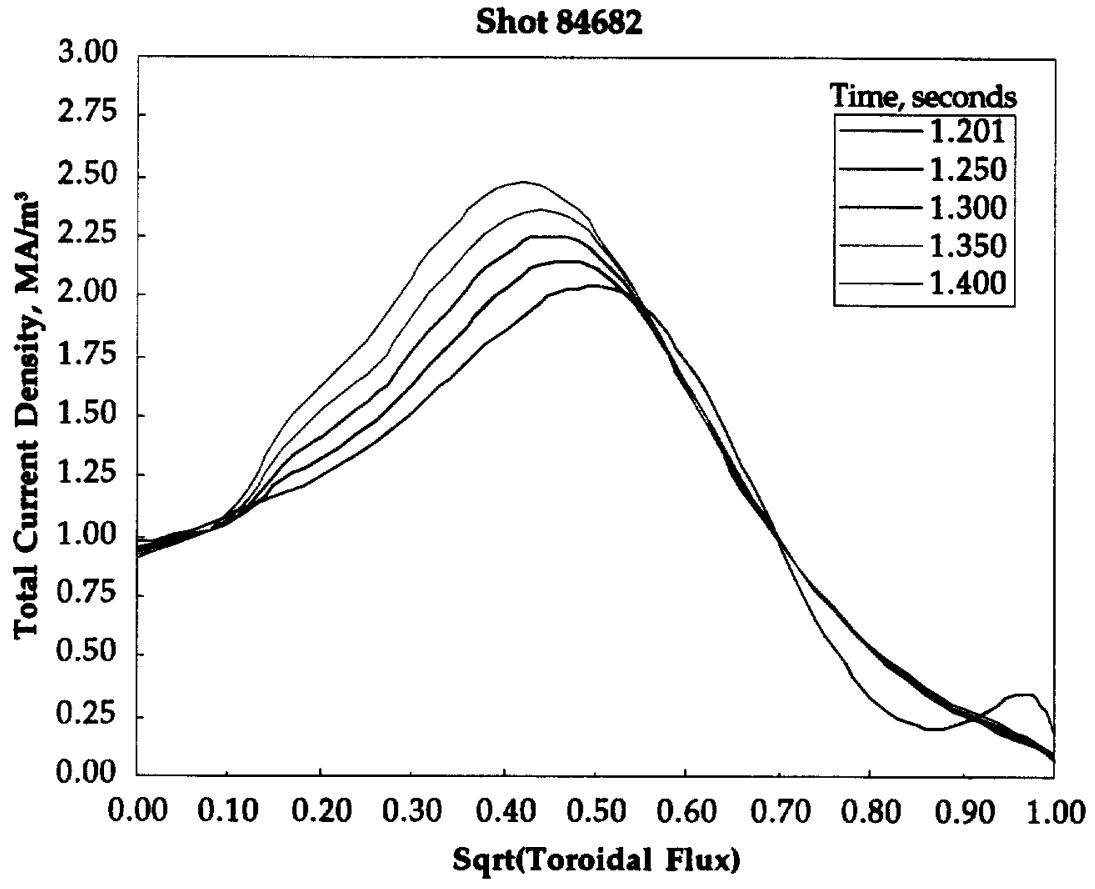


the improved orbit models and reviews in detail the physics that was corrected. The new neoclassical calculation will give smaller values than those rendered by current models and will generally agree with data, but these improvements were not yet incorporated into the version of TRANSP used in this study. There are error bars on the experimental data which result in TRANSP placing large error bars on the near-axis transport coefficients deduced from the experimental profiles. In fact, it is not uncommon for TRANSP to predict negative values of $\chi_{\mathrm{i}}$ in the central region!

The electron and ion thermal conductivities and the particle diffusivity deduced by TRANSP from shot 84682 are shown below in Figure III.1. This analysis was done at short intervals starting at 1.2 seconds, but approximately two hundred milliseconds of data were needed for TRANSP to start giving reliable data due to the fact that the Monte Carlo calculations of fast ion slow down times are this long and must come into equilibria before the results are meaningful. Therefore, profiles at 1.38 seconds were chosen for a self-consistent data analysis set. The particle diffusivity and the electron thermal diffusivity are well-behaved throughout the core, but the ion thermal diffusivity begins to fluctuate within the NCS region. If this entire $\chi_{i}$ profile is used, it gives unrealistic temperature profiles that decrease with time.

Figure III.1

TRANSP Transport Coefficient Profiles at 1.38 seconds

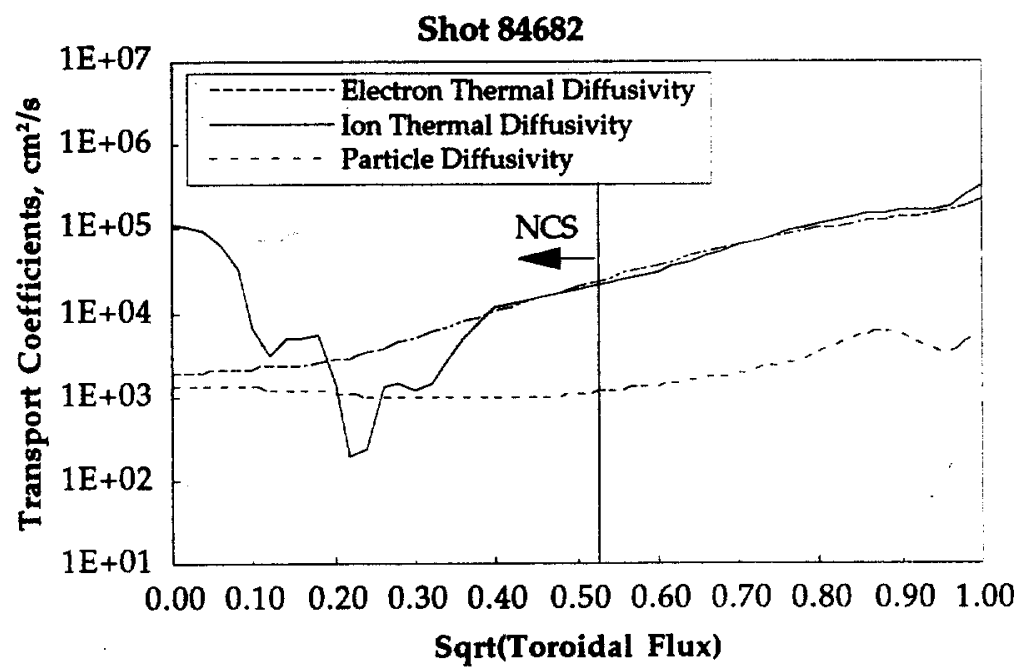

Consequently, $\chi_{\mathrm{i}}$ data from TRANSP was used in the positive shear region and a constant (trial) value of $\chi_{1}$ was assumed in the negative shear region. The criterion for chosing an acceptable trial value was that the on-axis ion temperature match the experimental value. A linear transistion of width $0.12 \sqrt{\psi}$ around the zero shear radius was made to join these two values, a transistion that was smoothed by CORSICA as shown in the adjusted profile in Figure III.2. Without that transition, there is a discontinuity in $\chi_{i}$, as shown by the unadjusted profile in Figure III.2. These composite profiles were used in CORSICA and the resulting profiles of temperature were matched against the actual profiles until a trial value was found that produced the best match.

Shown in Figure III. 3 are the resulting electron and ion temperature profiles overlayed with the experimental profiles. As can be seen from Figure III.3a, the unadjusted $\chi_{i}$ profile gives temperature profiles in reasonable agreement with the experimental profiles, but they are further improved in Figure III.3b by smoothing the abrupt transition as described above. Note that CORSICA closely replicates the electron temperature profiles, as it should, when using the TRANSP value of electron thermal diffusivity (which was determined from this very profile). This fact, along with the good agreement on ion temperature profiles, gives an independent check on CORSICA. 
Figure III.2

Comparison of Unadjusted and Adjusted $\chi_{i}$ at 1.38 seconds

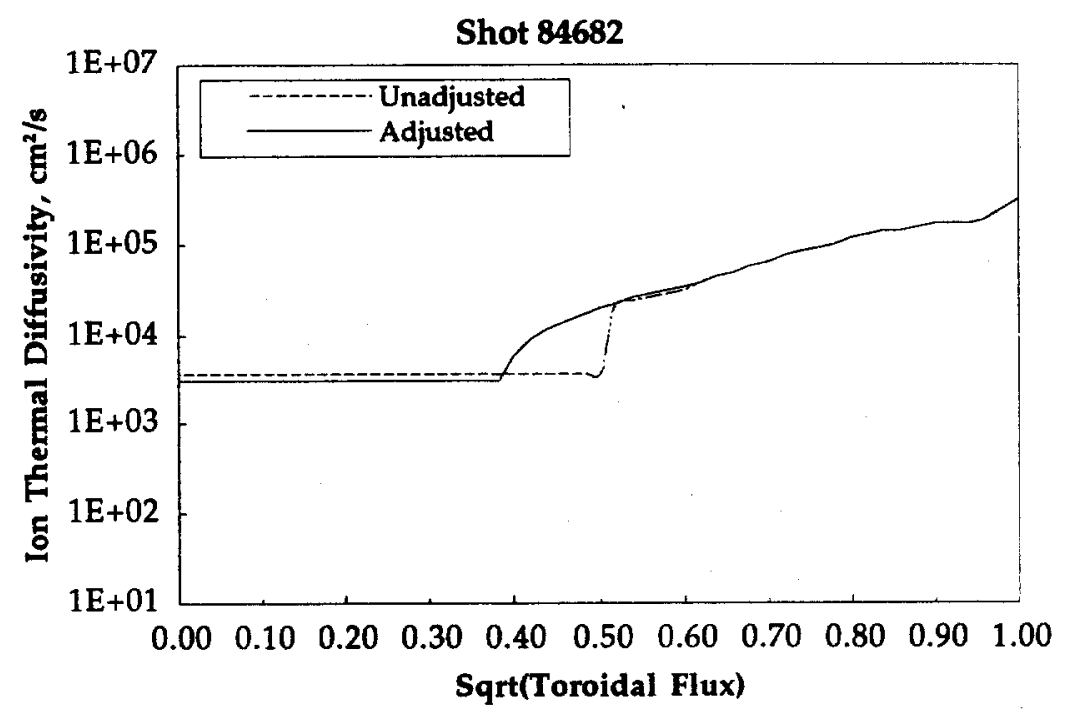

Figure III.3

Comparison of Resulting Temperature Profiles Using the $\chi_{i}$ Shown in Figure III.2 at 1.38 seconds

(a)

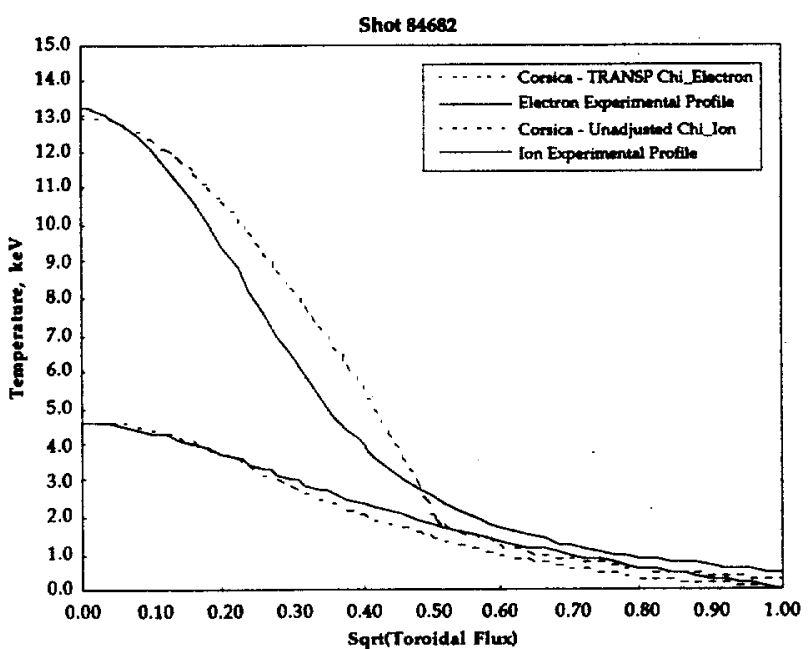

(b)

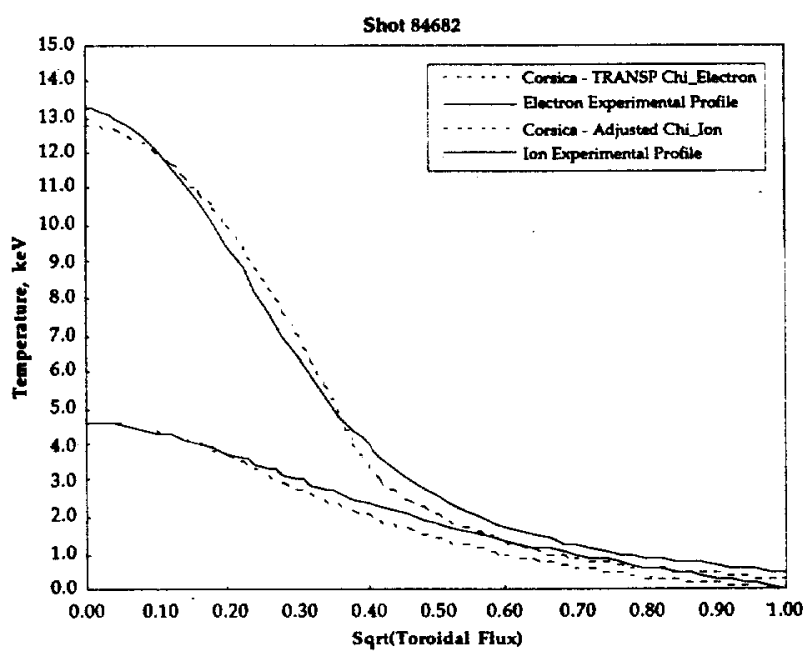

Complete profiles of $\chi_{i}, \chi_{e}$ and D at 1.38 seconds as determined from TRANSP, except for the ad hoc constant value for $\chi_{i}$ in the NCS region, have now been obtained. These profiles are the best estimate of the experimentally determined values using this methodology. With these in hand, comparisons can be made between these transport coefficient profiles and those used in CORSICA, which are the sum of the Chang-Hinton and RLW values. This is discussed in the next section.

The q-profile and total current density profiles shown in Figure III.4 are computed by CORSICA. These are generated using both the experimental pressure, which determines the diamagnetic current and field, and the calculated pressure, which uses the adjusted $\chi_{i}$ profiles shown in Figure III.2 and the TRANSP values of $\chi_{\mathrm{e}}$ and D shown in Figure III.1.

Now the methodology for generating composite transport coefficient profiles used in evolving the temperature profiles beyond 1.2 seconds can be described. For the NCS region, the portions of $\chi_{\mathrm{i}}$ and $\chi_{\mathrm{e}}$ 
constructed from TRANSP data at 1.38 seconds are used without apology, and they are used for all timesteps. It is assumed that the values there are fairly representative of the low transport coefficients during this time slice. In regions of positive shear, the RLW model is programmed to turn on if the critical gradient is exceeded, which it always does, and is added to the Chang-Hinton profile. Again, to avoid any step transitions, the zero shear position is found and these two profiles are linearly joined over a span of $0.12 \sqrt{\psi}$, centered about the point where magnetic shear is zero. This was done for all transport coefficients.

\section{Figure 111.4}

Safety Factor and Total Current Density Profiles with NCS Adjusted $\chi_{i}$ at 1.38 seconds

(a)

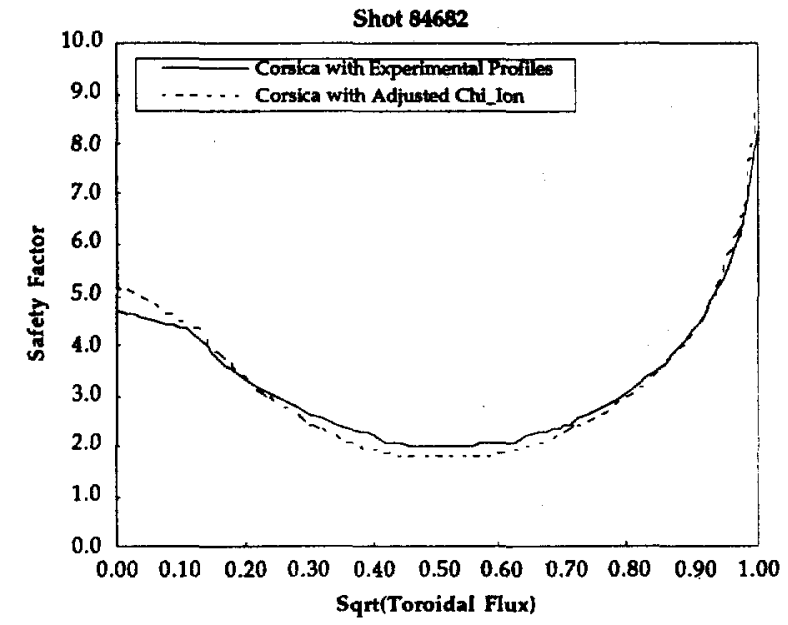

(b)

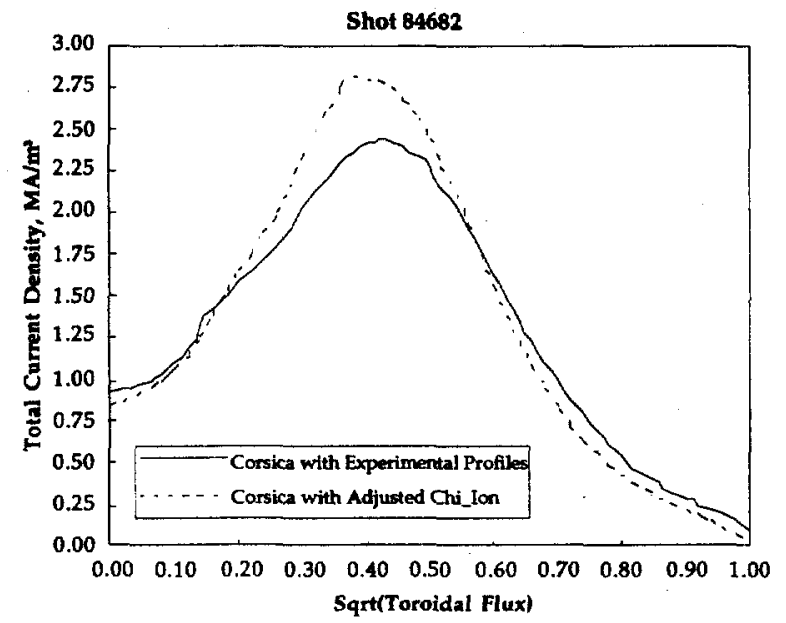

\section{RESULTS AND CONCLUSIONS}

The composite NCS/RLW model was evaluated by comparing its transport coefficients with the experimentally-derived profiles at 1.38 seconds. First, by using CORSICA, the Chang-Hinton neoclassical values were computed over all flux surfaces, then added the RLW portion in the positive shear region. The resulting transport coefficients are shown in Figures IV.1 below. At 1.38 seconds, the shear is zero at approximately $\sqrt{\psi}=0.52$ based on CORSICA's evaluation of experimental data, and the shear is zero at approximately $\sqrt{\psi}=0.43$ using the RLW transport model.

Figure IV.1a shows $\chi_{\mathrm{e}}$ as determined by the Chang-Hinton neoclassical formula in CORSICA. Note the erroneous rise towards the center of the plasma inside the NCS region. Outside of that region, the neoclassical value is 2-3 orders of magnitude lower than the (anomalous) experimental value, as given by the TRANSP curve. The RLW+Neo curve is our composite model. In the outer region, since the neoclassical contribution is negligible, the RLW mode accounts for the entire discrepancy between models and experiment at the zero shear point, and most of it as one progresses further out. The largest error is at the edge of the plasma, where gradients are much smaller and hence the impact is less important.

Similarly, Figure IV.1b shows $\chi_{i}$ as reconstructed in the same three ways, except that the TRANSP value has been modified as in Figure III.2. Again, the RLW model matches the experimental data near the zero shear portion of the curve, and underestimates it progressively as the edge of the plasma is approached. In Figure IV.1c the RLW model overestimates the particle diffusivity by an order of magnitude. Despite this disagreement, it is important to note that the RLW correction to neoclassical values is substantial, and likely to give reasonable predictions of profiles from initial data. 
(a)

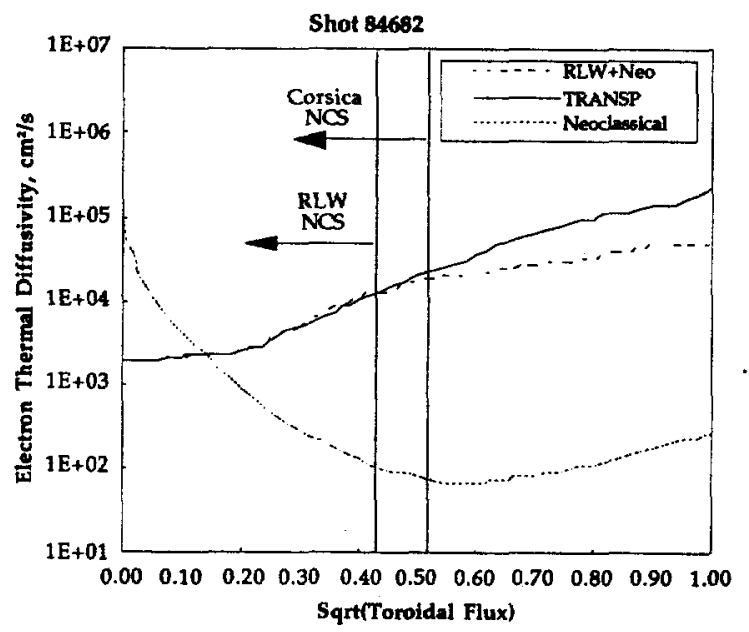

(b)

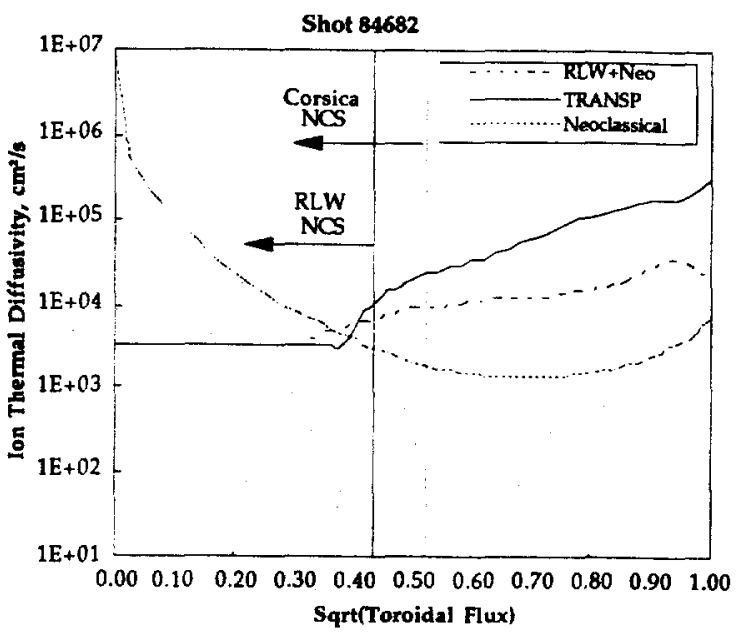

(c)

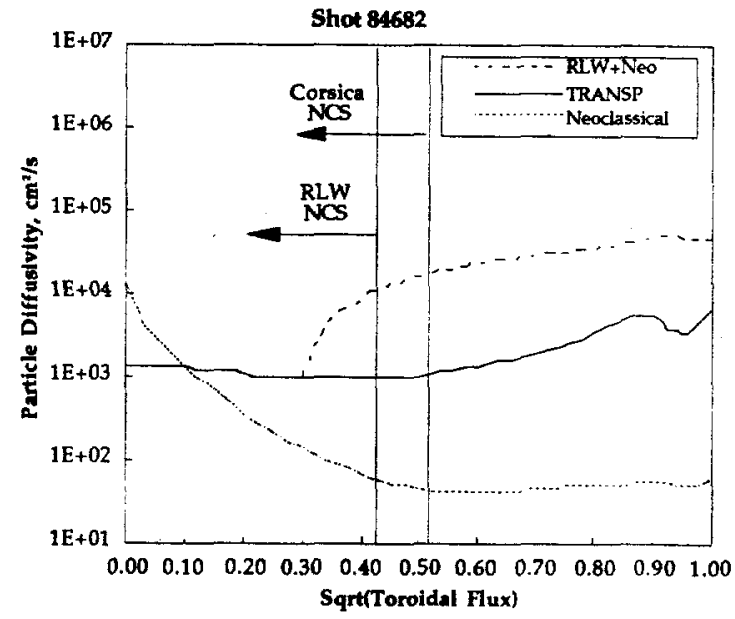

To illustrate that point, the experimental and calculated profiles of the ion and electron temperatures at 1.38 seconds are shown in Figure IV.2. Here it is seen that, while the profile differences are noticeable, the model nonetheless reproduces its major features and the absolute values are not greatly in error. For general predictive purposes, these features may suffice if one is looking for broad trends.

Figure IV.3 shows a comparison between experimental and RLW-generated profiles of the safety factor and total current density. The safety factor is a good match. The current density peak is slightly misplaced spatially, but overall has a reasonably close shape.

If the NCS RLW coefficients in Figure IV.1 are used for all time steps, CORSICA will evolve electron and ion temperature profiles. Starting at 1.2 seconds, Figure IV.4 shows the resulting profiles in 50 millisecond intervals where the RLW+Chang-Hinton models are used within the positive shear region. The profiles are smooth, well-behaved, but unfortunately show the on-axis temperature increasing then decreasing with time. This can perhaps be explained by examining Figure IV.1c. The RLW model significantly overshoots the experimentally-derived value for particle diffusivity. Even though the experimental density profiles were used in this analysis, $D$ is still used to determine the convection velocity in the Boltzmann-derived energy equation employed by CORSICA. An overestimated particle diffusivity would mean an overestimated convection term, hence falling 
temperature profiles. As discussed in the introduction, the RLW model acknowledges a constant of proportionality between $\mathrm{D}$ and $\chi_{\mathrm{e}}$, namely, $\mathrm{D}^{\mathrm{AN}}=\alpha \chi_{\mathrm{e}}^{\mathrm{AN}}$, where $\alpha$ was initially set equal to 1.0 . In a future study, this parameter could be adjusted until the RLW particle diffusivity matched the experimentally-derived particle diffusivity and the profiles could be re-evolved to see if the on-axis temperatures increase, as expected, and to see whether or not the profiles maintain their shape.

\section{Figure IV.2}

\section{Comparison of Electron and Ion RLW Temperature Profiles at 1.38 seconds}

(a)

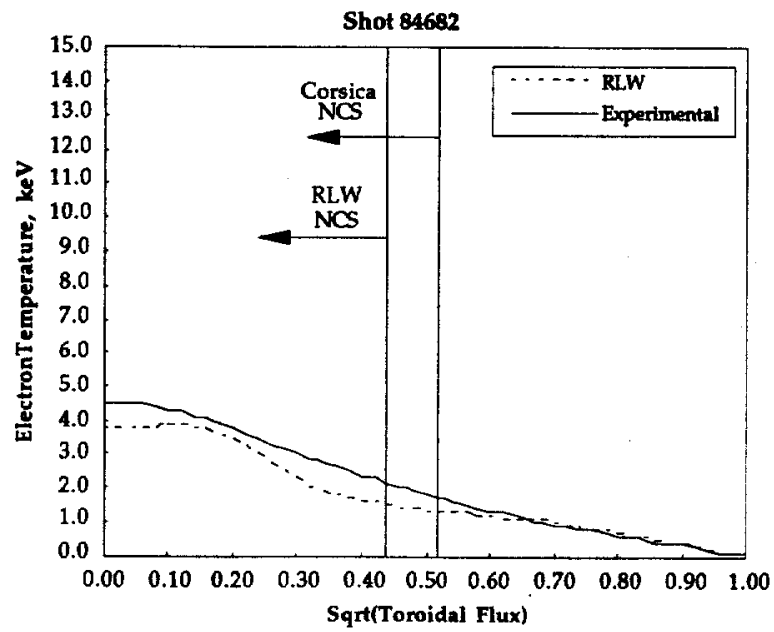

(b)

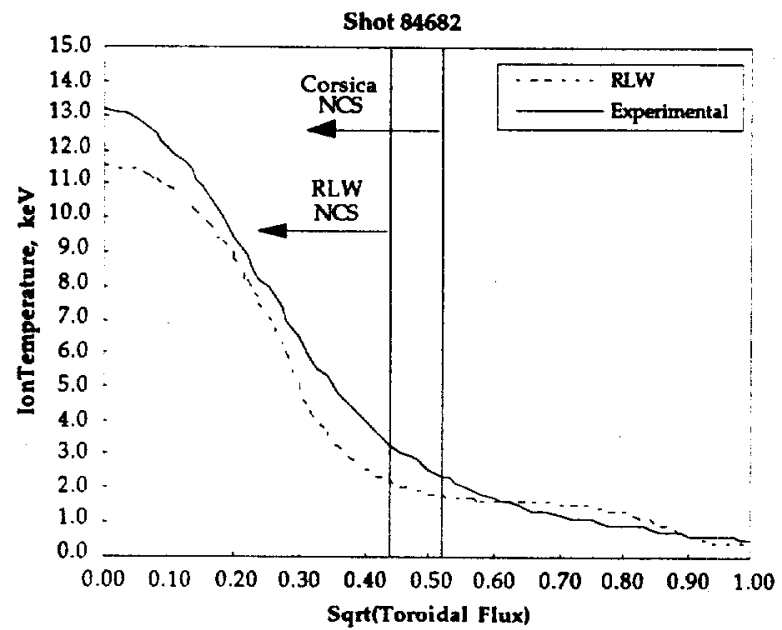

Figure IV.3

Comparison of Safety Factor and Total Current Density RLW Profiles at 1.38 seconds

(a)

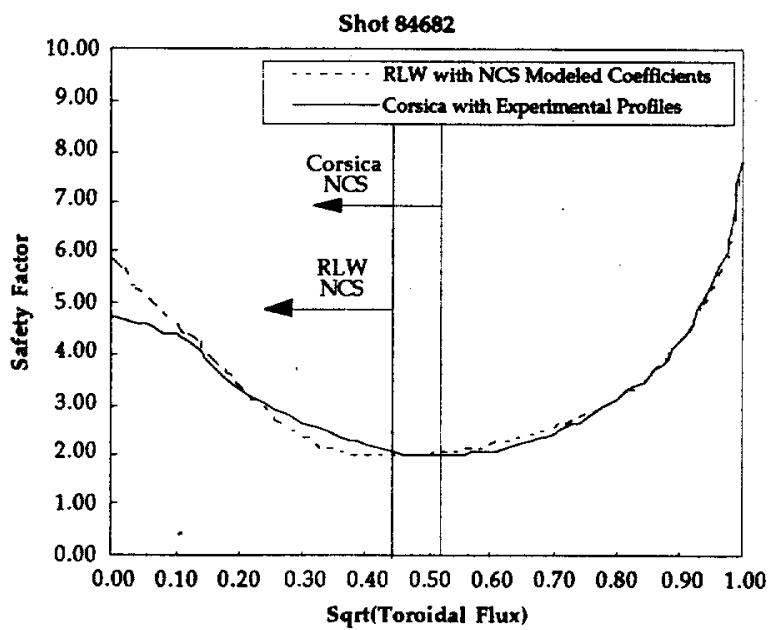

(b)

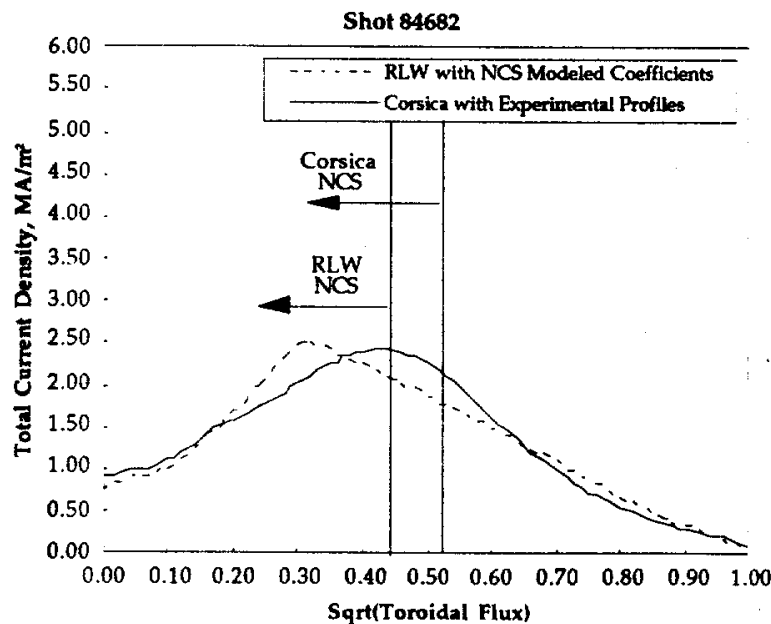

There is a significant amount of additional follow-on work which could be done. First, a credible NCS transport model is needed. From experimental data quoted earlier it is clear that instabilities are largely suppressed in that region, hence it might be expected that a correct neoclassical model would suffice. The recent work [11] described earlier can provide that model. Given such a model, its marriage with the RLW transport model could further improve the model developed here. Secondly, 
Figure IV.4

(a)

RLW Electron Temperature Profiles

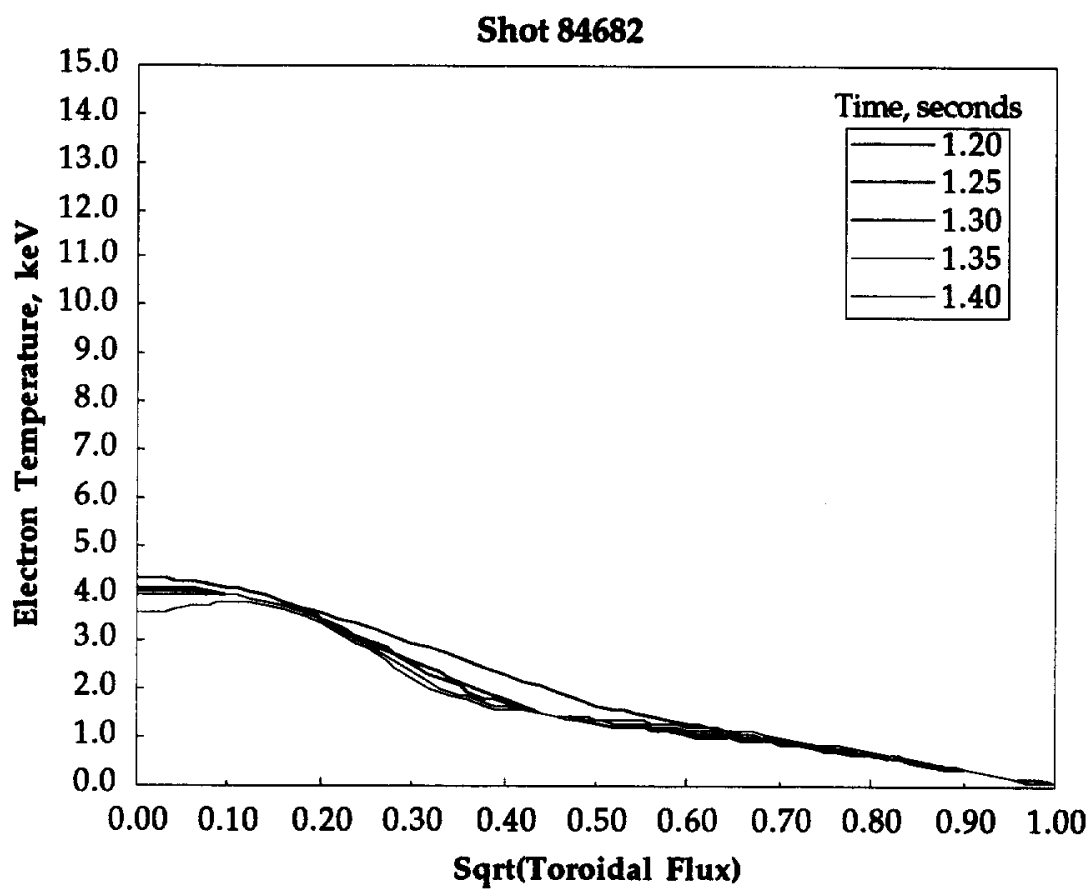

(b)

RLW Ion Temperature Profiles

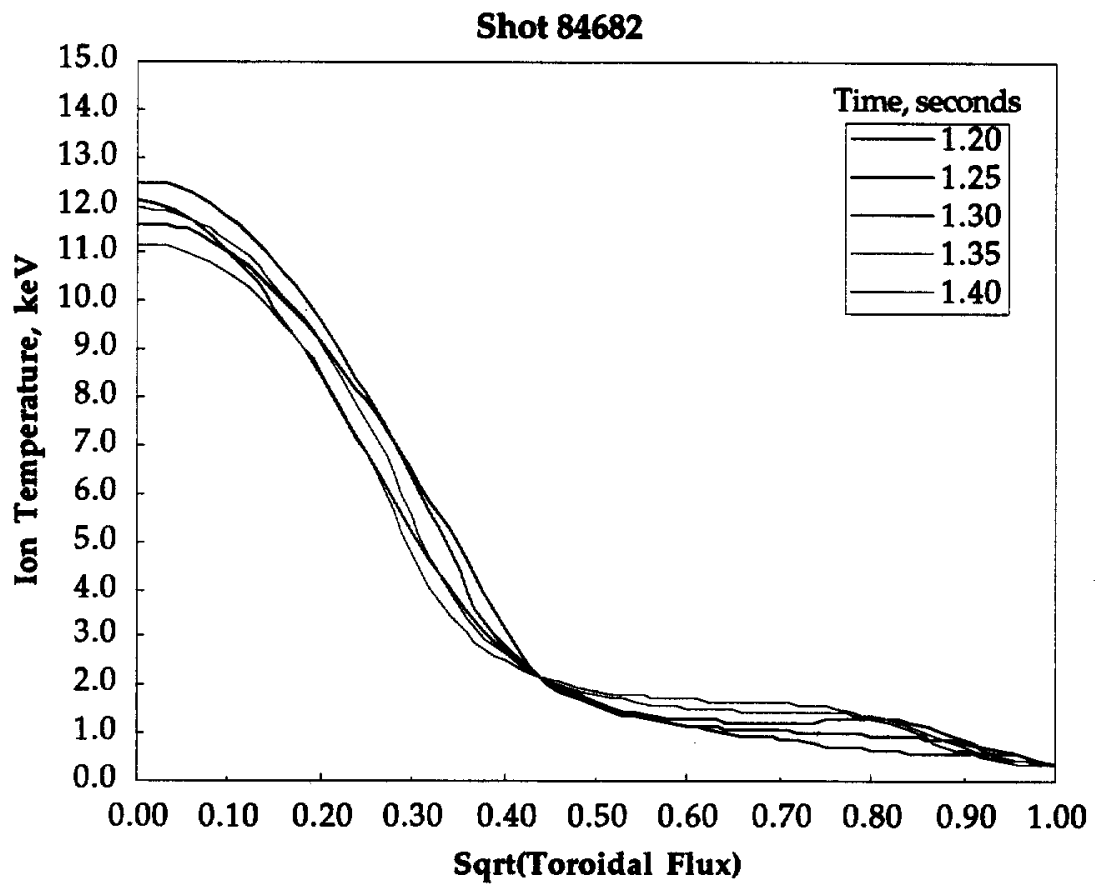


additional models in the positive shear region could be incorporated into CORSICA and would provide a predictive capability that would be very important to current research. Finally, with at least an initial model in hand, one can proceed to evaluate means for optimizing the NCS modes in DIII-D using neutral beam and other heating and current drive sources to optimize the location of the zero shear surface, the timing and aiming of beams, and other adjustments that might lead to better performance.

\section{Acknowledgements}

The authors wish to thank MFE staff at LLNL, particularly Jim Crotinger and Don Pearlstein for assistance with CORSICA and Basis scripts.

\section{References}

[1] P. Rebut, P. P. Lallia, and M. Watkins, Plasma Physics and Controlled Nuclear Fusion Research, Nice, France (IAEA, Vienna, 1989), 2, 191 (1988).

[2] P. Rebut, M. Watkins, D. Gambier, and D. Boucher, Physics of Fluids B 3, 2209 (1991).

[3] D. Boucher and P. Rebut, IAEA Technical Committee Meeting (Montreal 1992).

[4] C. Chang and F. Hinton, Phys. Fluids 29, 3314 (1986).

[5] F. Levinton et al., "Improved Confinement with Reversed Magnetic Shear in TFTR," Physics Review Letters 75, 4417 (1995).

[6] E. Straight et al., "Enhanced Confinement and Stability in DIII-D Discharges with Reversed Magnetic Shear," Phys. Review Letters 75, 4421 (1995).

[7] B. Rice, T. Taylor, T. Casper, C. Forest, H. Ikezi, L. Lao, E. Lazarus, M. Mauel, B. Stallard, and E. Strait, "The Formation and Evolution of Negative Central Magnetic Shear Current Profiles on DIII-D," Plasma Physics and Controlled Fusion 38, 869 (1996).

[8] B. Rice et al., "Demonstration of High-Performance Negative Central Shear Discharges in the DIII-D Tokamak," Phys. Plasmas 3, 1983 (1996).

[9] B. Rice et al., "Observations of Enhanced Core Confinement in Negative Magnetic Shear Discharges with an L- mode Edge on DIII-D," Nuclear Fusion 36, 1271 (1996).

[10] L. Lao et al., "Rotational and Magnetic Shear Stabilization of Magnetohydrodynamic Modes and Turbulence in DIII-D High Performance Discharges," Phys. Plasmas 3, 1951 (1996).

[11] Z. Lin, W. Tang, and W. Lee, "Neoclassical Transport in Enhanced Confinement Toroidal Plasmas," Phys. Review Letters 78, 456 (1997).

[12] J. Wesson, Tokamaks, Clarendon Press, Oxford, Chapter 4 (1987).

[13] J. Crotinger et al., Bull. Am. Phys. Soc. 38, 2016 (1993).

[14] R. Hawryluk, in Physics of Plasmas Cose to Thermonuclear Conditions (COPPI, B. et al., Eds), Vol. 1, CEC, Brussels, 19 (1980). 


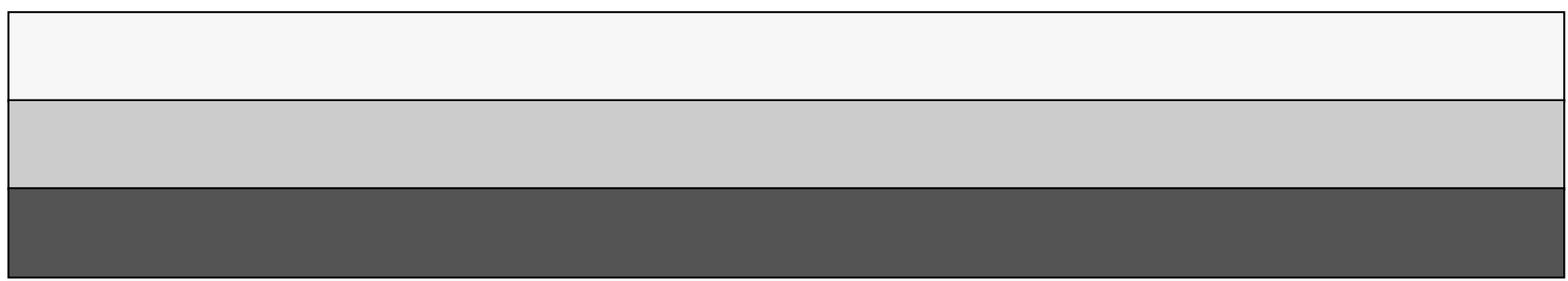

